\title{
Concept for an Onboard Integrated PNT Unit
}

\author{
R. Ziebold, Z. Dai, T. Noack \& E. Engler \\ Institute of Communications and Navigation, German Aerospace Centre (DLR), Neustrelitz, Germany
}

\begin{abstract}
A robust electronic position, navigation and timing system (PNT) is considered as one of the core elements for the realization of IMO-s (International Maritime Organization) e-Navigation strategy. Robustness can be interpreted as the capability of an integrated PNT system to provide PNT relevant data with the desired accuracy, integrity, continuity and availability under consideration of changing application conditions and requirements. Generally an integrated PNT system is a composite of service components - like GNSS, Augmentation Systems and terrestrial Navigation Systems - and an on-board integrated PNT Unit, which uses the available navigation and augmentation signals in combination with additional data of sensors aboard to provide accurate and robust PNT information of the ship. In this paper a concept of such an onboard integrated PNT Unit will be presented, which is designed to fulfill the specific user requirements for civil waterway applications. At first, the user requirements for an integrated PNT Unit will be overviewed. After that, existing integrity monitoring approaches will be analyzed. Finally, a first integration scheme for an integrated PNT Unit will be presented with a special focus on the internal integrity monitoring concept.
\end{abstract}

\section{INTRODUCTION}

The maritime integrated PNT System (Figure 1) is the sum of satellite-based, ashore and aboard components. The integrated use of these components enables the accurate and reliable provision of position, navigation and timing data to all maritime applications.

Position fixing systems are identified as one strategic key element of e-navigation [1]. Existing and future Global Navigation Satellite Systems (GNSS) like GPS, GLONASS and GALILEO are fundamental infrastructures for global positioning. Additionally, terrestrial services are used or considered as candidates to improve the positioning performance (augmentation services: e.g. IALA Beacon DGNSS, RTK) or to ensure the backup functionality (backup services: e.g. e-LORAN, R-Mode) respectively to GNSS. Due to their interoperability and compatibility these systems can be used alternatively or complementary for positioning, navigation and timing.

The International Association of Marine Aids to Navigation and Lighthouse Authorities (IALA) has introduced the term "integrated PNT device" to describe the on-board part of maritime, integrated PNT system. In [2] the integrated PNT device is described as "a device using any available IMO recognized radio navigation systems simultaneously to provide the best electronic position fix for the ship". Following this definition, the outlined objective of the PNT device is focused on the provision of position information to different applications. Several performance standards for shipborne GNSS and DGNSS receivers were developed and approved by IMO in the last decade: GPS [3], GLONASS [4], DGPS and DGLONASS [5], combined GPS/GLONASS [6], and GALILEO [7]. A logical consequence of this standardization process could be the preparation of a new performance standard for a multi-system radio navigation receiver as core element of the on-board part of the PNT System (Figure 1). A more generally admitted approach can be achieved by the introduction of the PNT Unit.

The on-board PNT Unit aims at the provision of position, navigation and timing data in accordance with specified performance requirements, which change during berth to berth navigation. The core of the on-board PNT Unit is a value-added processing system using available radio navigation systems and services in combination with on-board sensors for accurate and reliable PNT-data provision. The onboard PNT Unit is on the one hand part of the integrated PNT system and on the other hand part of the on-board Integrated Navigation System (INS). 


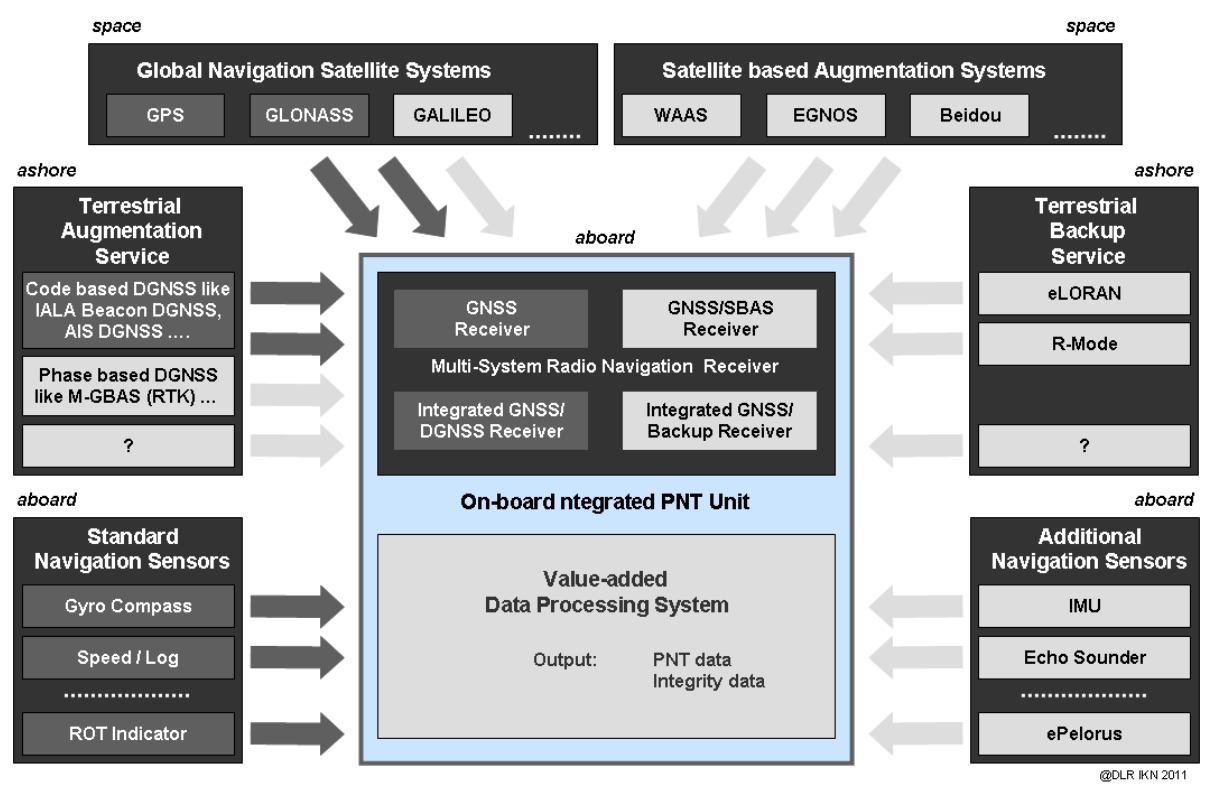

Figure 1. Integrated PNT System (dark grey: standard, light grey: considered options)

Due to user needs such as "Indication and Improvement of Reliability" and "Alarm Management” [1] identified within the framework of the eNavigation process, a more general approach for the on-board part of the integrated PNT system shall be aimed. Reasons for this perspective are on the one hand the need for redundancy to improve the robustness of PNT-information and to enable the assessment of accuracy by suitable integrity monitoring functions. On the other hand the type of implementable redundancy (equipment, different measurement methods, over determined systems, alternative applicable techniques) specifies the potential of error detection, identification and mitigation.

\section{TECHNICAL REQUIREMENTS AND SENSORS}

\subsection{Technical requirements}

In [8] the aim of GNSS is described as a system to provide worldwide position, velocity and time determination for multimodal use. An operational requirement indicates the tasks of shipborne GNSS devices in the provision of position, time, course and speed over ground. But in Appendix 2 and 3 of the same document minimum maritime user requirements are only given for horizontal position. Otherwise the use of the item Electronic Position Fixing System (EPFS) in [9] implicates that the scope of GNSS is rather the provision of position data than the provision of PNT data.

The analysis of these documents shows the necessity to clarify and define the extent of PNT data, which should be delivered by a maritime PNT Unit. In a preliminary design, the following parameters should be considered:
1 Position: It mainly contains the longitude and latitude for maritime navigations. Because vessels can usually be found close to the sea level, the height information is usually not provided as standard output parameter.

2 Under keel clearance (UKC): Instead of the height information, the UKC is the relevant maritime output parameter. It is defined as the distance between the lowest point of the ship (e.g. the keel) and the ground of the sea.

3 Velocity: The magnitude and direction of a velocity vector can be described by Speed over Ground (SOG) and Course over Ground (COG). Because of their physical principles, speed sensors like e.g. electro-magnetic logs can only measure the speed through water (STW), and therefore STW is also a parameter which a PNT Unit could deliver.

4 Attitude: Generally the orientation of the ship in the horizontal plane is reported. Here one needs to distinguish between the orientation with respect to the true north (true heading) and with respect to the magnetic north (heading). For future applications, the other attitude angles, namely roll and pitch, could also be required.

5 Timing: UTC time needs to be delivered.

After the clarification of the PNT output parameters, further additional requirements on the PNT Unit will be discussed in the following.

In [1], the robustness of all e-navigation systems is requested. In order to fulfill this requirement, a definition of robustness needs to be given. We interpret robustness as the ability of a system to provide the output data according to their specification under changing application conditions and in cases of external disturbances (interferences, jamming, atmospheric influences). The robustness shall therefore be applicable to the realization of the basic functional- 
ity (output data with required accuracy) and integrity functionality.

In [1] it is furthermore stated, that requirements for redundancy, particularly in relation to position fixing systems, should be considered. Redundancy in a general meaning can be seen as the provision of an alternative system to support critical system functionalities. Within the Recommendation R-129 on GNSS Vulnerability and Mitigation Measures [10] IALA has given a classification of alternative navigation systems in relation to their aims:

1 A redundant system provides the same functionality as the primary system, allowing a seamless transition with no change in procedures.

2 A backup system ensures continuation of the navigation application, but not necessarily with the full functionality of the primary system and may necessitate some change in procedures by the user.

3 A contingency system allows safe completion of a manoeuvre, but may not be adequate for longterm use.

For the introduction of additional sensors to the integrated PNT Unit, this classification scheme needs to be considered.

\subsection{Standard sensors for the provision of PNT data}

According to the carriage requirement demanded by the IMO Safety of Life at Sea (SOLAS) convention [11], following sensors (see Table 1) should be used in maritime applications. An overview of the sensors and a discussion of the related standards can be found in [12]. Therefore here only a table with the list of standard sensors with typical output data and realizations are given.

TABLE 1. STANDARD PNT SENSORS

\begin{tabular}{|l|l|l|}
\hline Sensor & Output & Typical realization \\
\hline \multirow{2}{*}{ Speed log [13] } & $\begin{array}{l}\text { Speed through water } \\
\text { (STW) }\end{array}$ & $\begin{array}{l}\text { Electromagnetic } \\
\text { logs }\end{array}$ \\
\cline { 2 - 3 } & $\begin{array}{l}\text { Speed over ground } \\
\text { (SOG) }\end{array}$ & Doppler logs \\
\hline $\begin{array}{l}\text { Compass [14] } \\
\text { [15] }\end{array}$ & $\begin{array}{l}\text { True heading } \\
\text { Magnetic heading }\end{array}$ & Gyrocompass \\
\hline $\begin{array}{l}\text { Electronic Posi- } \\
\text { tion Fixing Sys- } \\
\text { tem (EPFS) }\end{array}$ & $\begin{array}{l}\text { Position } \\
\text { Speed over Ground } \\
\text { (SOG) }\end{array}$ & $\begin{array}{l}\text { GPS/GLONASS } \\
\text { DGPS/DGLONASS } \\
\text { receiver and antenna } \\
\text { (COurse over ground } \\
\text { Time }\end{array}$ \\
\hline $\begin{array}{l}\text { Transmitting } \\
\text { Heading Device } \\
\text { [16] (THD) }\end{array}$ & Heading & $\begin{array}{l}\text { GNSS multi- an- } \\
\text { tenna system }\end{array}$ \\
\hline $\begin{array}{l}\text { Echo sounder } \\
\text { [17] }\end{array}$ & UKC & Sonar \\
\hline $\begin{array}{l}\text { Rate of Turn } \\
\text { Indicator [18] } \\
\text { (ROTI) }\end{array}$ & ROT & Mechanical Gyro \\
\hline
\end{tabular}

3 ACCURACY ASSESSMENT AND INTEGRITY MONITORING

\subsection{Integrity Definition}

One of the key tasks of an integrated PNT Unit is the additional provision of integrity messages to the user. Within the specification of requirements for future GNSS [8] IMO has given the following definition of in integrity: The ability to provide users with warnings within a specified time when the system should not be used for navigation. Within that definition the alert limit specifies the applicable threshold differing between an unusable or unusable system. The time to alarm (TTA) describes the acceptable time duration between occurrence of an intolerable error and the provision of the related integrity message. The remaining integrity risk (IR) specifies the tolerable probability, that the assessment of accuracy by integrity monitoring is erroneous. This definition implies an integrity monitoring process wherein the accuracy of the given navigation parameter needs to be estimated in real time and compared against a given threshold (alert limit). In other words, an error estimation of all navigation parameters needs to be performed onboard a vessel.

Integrity monitoring as it is defined for Integrated Navigation Systems (INS) consists of the following three sequential steps [19]:

- Plausibility check

The plausibility check tests whether the sensor raw data or derived navigational result falls into predefined value range. The plausibility check is normally carried out at receiver side in order to test the usability of the sensor data.

- Validity check

The validity is tested by comparing the sensor data or derived navigational results with formal and logical criteria as well as by checking the correctness of the data format. The validity check is normally carried out at the sensor site in order to ensure the proper operation of the sensor.

- Compatibility check

Once a specific parameter can be provided by more than one sensor, different sensor data can be compared to test the compatibility. A significant discrepancy between different sensor data implies the failure of at least one of these sensors. The upper bound for deviation should be defined either a priori or in real-time according to the previous measurements. The compatibility test should be carried out before sending the sensor data to integration algorithm.

The primary aim of plausibility, validity and compatibility checks is the detection of errors. The assessment of accuracy requires the implementation of suitable integrity monitoring functions. 


\subsection{Existing Integrity Monitoring approaches}

In this section existing integrity monitoring approaches defined for maritime navigation will be described and the seen gaps will be discussed.

\subsubsection{Integrity monitoring for GNSS}

\subsubsection{Receiver Autonomous Integrity Monitoring (RAIM)}

RAIM is a technique, which can be applied in GNSS receivers to assess the integrity of navigation signals [8], if more than 5 GNSS signals are tracked [8]. RAIM has two-fold tasks, first is to check the occurrence of a failure, and second is to identify erroneous satellites. RAIM can be done by using only the incoming measurement of current epoch or employing also measurements of previous epochs. The former approach needs the redundant measurement, and the performance is dependent on the number of satellites in view. In [20], several classic "snapshot" algorithms are introduced.

For a maritime GNSS receiver a RAIM is requested [21], however, neither the algorithm is specified nor it is defined how the user should react on the three possible outputs: safe, caution, and unsafe. Referring to a "snapshot" RAIM, the a priori knowledge of the observation errors is needed by all existing algorithms. A proper determination of this term for maritime navigation is an open task.

\subsubsection{IALA Beacon DGNSS}

GNSS integrity-monitoring services are usually part of augmentation services which also provide DGNSS corrections. The reason for this shared activity is the similarity of the infrastructure required for DGNSS and integrity monitoring.

The IALA Beacon Differential GNSS service is a standardized technique for maritime use [22]. The implemented integrity monitoring assesses primarily the integrity of the service itself, but can also include parts of GNSS assessment. At reference station different thresholds are applied to the observed HDOP or determined range and range rate corrections to estimate the usability of service or single GNSS signals. Additionally the IALA Beacon DGNSS is equipped with one or more integrity monitoring stations operating as virtual user at known location. Only in case that the observed DGNSS position error is below the allowed error threshold, the IALA Beacon DGNSS distributes a flag bit to indicate the usability of the service. The health status of a satellite is provided indirectly by embedding the "do not use" flag in the transmitted PRC and RRC for an unhealthy satellite.

Spatial decorrelation of provided range and range rate corrections, differences in satellite visibility between station and user sites, and user specific reception conditions (multipath, interferences) are the main causes for discrepancies between the estimated and real DGNSS positioning performance.

\subsubsection{Maritime GBAS (RTK)}

The preliminary integrity monitoring of the phase based Maritime GBAS [23] follows the IALA Beacon DGNSS concept. Hence an integrity monitoring station (IM) is installed in the service area (ca. 10$20 \mathrm{~km}$ ) of the reference station (RS). At both stations integrity monitoring procedures are processed in three steps. In the first step the RS and the IM evaluate the quality of the received GNSS signals on the basis of quality parameters like phase and code noise. In a second step a GNSS RAIM based position determination is realized. The results of both steps are compared with pre-specified thresholds to assess the usability of single GNSS signals as well as the usability of the service of RS and IM station.

Afterwards the signals without abnormalities from the RS and IM are used to calculate the IM position by carrier phase based differential techniques (RTK). The position error is derived by comparison of the computed with the exactly known position of the IM. If the accuracy requirements of IMO for port vessel operation are fulfilled, the service can be considered as usable.

Finally the M-GBAS logically combines all gathered integrity information at RS and IM site gained in the previous steps to generate the RTCM 3 messages for the provision of augmentation data and related integrity data (message 4083).

\subsubsection{Integrity monitoring for INS}

An INS offers integrated and augmented functions to support system tasks like collision avoidance, route planning and route monitoring. Currently an INS is not a mandatory system, but if an INS is installed onboard a vessel it is accepted monitoring is considered as an intrinsic function of the INS. The currently valid INS standard is based on IMO resolution MSC.86 (70) [24] and is specified within the IEC-61924 standard [19]. A task oriented concept is already introduced in a new resolution MSC.252 (83) [25], but the specification within the related IEC standard is not yet published. Therefore our analysis is based on IEC-61924 standard only. In IEC61924 standard, plausibility, validity and compatibility check approaches are introduced. IEC-61924 standard suggests the compatibility check for the following navigational parameters.

1 Position: comparison with a second EPFS; using RAIM GNSS function; Dead Reckoning (DR) using the ship's heading and speed measuring device

2 Heading: comparison with a second heading sensor and a course over ground sensor

3 SOG: comparison with a second SOG sensor, with speed through water sensor and with SOG from the EPFS (GNSS) 
4 Time: comparison with a second time sensor and with the internal INS clock

5 UKC: comparison with a second depth sensor and with data derived from ships position and electronic navigational charts (ENC)

\subsection{Demand on specification and development}

The Position, as the most important PNT information, currently is measured by only two separate receiver/antenna GNSS devices. Integrity monitoring is restricted to a comparison of the positions determined by these two receivers. Due to the same measurement technique, system and propagation errors of both GNSS devices underlie the similar error in the measurement and position domain.

An estimation of the actual position error is currently not performed onboard a vessel. Indirectly it is assumed, that the horizontal position error is $<100$ $\mathrm{m}$, when using GNSS standard positioning service, and $<10 \mathrm{~m}$, when using IALA Beacon DGNSS service.

Although positioning accuracy requirement on GNSS are specified with respect to different operational areas [26][8], the integrity monitoring is currently performed by using fixed or user selectable thresholds. In order to use these areas for future integrity monitoring applications, these areas and the intersection from one area to another need to be clearly specified in an appropriate way (e.g. in ENC charts).

Furthermore integrity specifications for the other PNT parameter (e.g. SOG, COG, time) besides position have to be specified. The necessity of an opera- tional area dependent accuracy and integrity specification for these parameters needs to be discussed

Analyzing the existing integrity monitoring approaches with respect to the identified user needs [1] a demand on the development of an enhanced integrity monitoring for all relevant PNT data within an integrated PNT Unit can be deduced. Such a PNT Unit should use sensor and data fusion methods to ensure the provision of PNT output data with the desired accuracy and to ensure an overall integrity monitoring for these output data. For both functionalities a higher degree of standardization is desired in order to achieve comparable results for their harmonized application.

\section{PROPOSED ARCHITECTURE OF INTEGRITY MONITORING IN PNT UNIT}

For a PNT Unit, integrity monitoring can be carried out in three sequential steps. The first step is an individual sensor data test. The second step is the compatibility test of similar data from different sensors. The third step is the fault detection and identification in the integration algorithm. A general integrity monitoring approach is depicted in Figure 2.

\subsection{Integrity monitoring for GNSS}

Actually within an INS, position integrity monitoring is performed by comparing the calculated position with a second EPFS, by using RAIM GNSS function and by using DR technique. Possible improvements in a PNT Unit will be elaborated as follows.

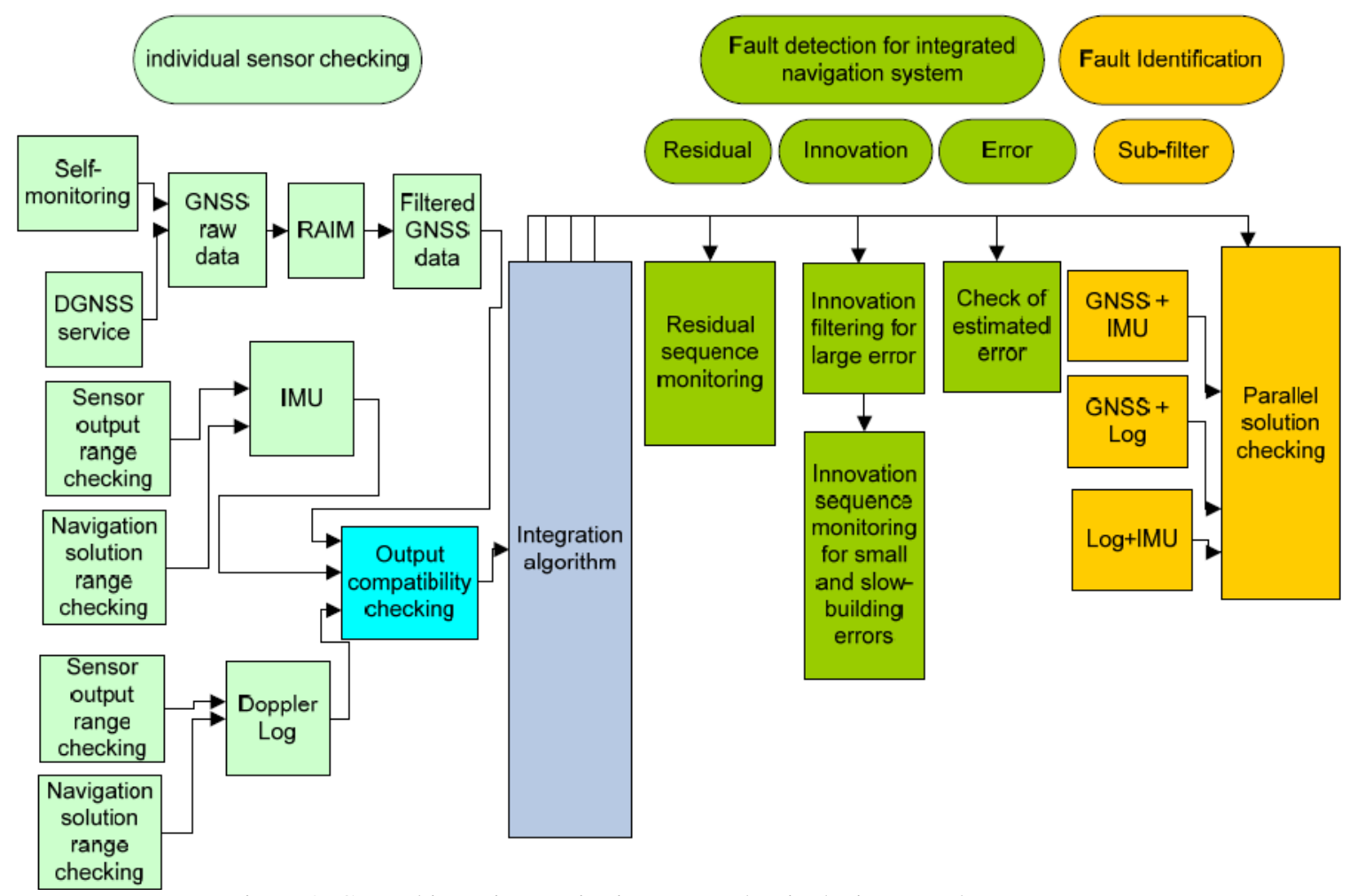

Figure 2. General integrity monitoring approaches in the integrated sensor system 


\subsection{Compatibility check for redundant GNSS systems}

GNSS redundancy can be achieved using a secondary GNSS device, using multiple civilian frequencies and using multiple GNSS constellations.

Once the major GNSS device (antenna or receiver) is out of use, the second GNSS device can fully take the function of the major GNSS device. However, the redundant GNSS device is also affected by the errors related to the radio signal. In this sense, the significance of a redundant GNSS device is reflected during the internal failure of the major GNSS device.

Modern GNSS satellites will facilitate more than one signal. Civilian code data will also be encoded into the carrier signal besides L1 signal at future GNSS satellites. The additional civilian code data will offer the same functionalities like the SPS service. Due to different carrier signals, the other carrier signals might not suffer from the same interference or jamming or propagation effects as L1 signal. Also, the channel failure (loss of lock or cycle-slips, etc.) for L1 signal might not occur simultaneously on the other frequencies. Nevertheless, errors due to space atmosphere and signal propagation will influence all the carrier signals of a satellite. Hardware failure of receiver or antenna might also challenge the reception of all carrier signals.

Two or more full-operational GNSS constellations could serve as redundancy for each other, as they realize same functions in maritime navigation as specified in [26]. However, GLONASS and GALILEO are not yet fully operational. Future GNSS receivers and corresponding antennas allow the reception and processing of multiple GNSS signals, however, a hardware failure can cause the loss of all GNSS signals.

\subsection{Compatibility check with backup systems}

Systems like e-Loran (enhanced Long Range Navigation) or R-mode (Ranging mode) facilitate the functions for positioning. The e-Loran system can also be used for time determination, so that these systems could serve as backup for GNSS [27]. Compared to GNSS, e-Loran signals are transmitted at lower frequency with higher power and hence it is not easy to be jammed especially not by the same GNSS jammers. It relies on the radio signal propagated over ground and hence does not suffer from the same errors in the propagation path from sky like GNSS. So the future of e-Loran as a terrestrial backup for GNSS with a large coverage area is currently an open question. Also, the fulfillment of the future maritime requirements with respect to the accuracy is an issue.

In [2], the R-Mode is seen as a possible novel variant of positioning technique using terrestrial signals. The idea is to use existing communication channels and append their functionality by sending an additional timing signal. From the time difference between signal transmission and reception, the ship should be able to determine its position. The advantage of this idea would be that at least partially existing infrastructure could be used. Currently this is still only an idea, where the proof of concept needs to be shown.

\subsection{Compatibility check with contingency system}

The DR is a frequently-used technique to predict the position using SOG and COG information. In maritime navigation, COG information is usually approximated by compass. DR is independent of the radio signals and hence still works during GNSS outage. In a sensor fusion system, DR is already an implicit function and does not need to be separately implemented.

Another contingency system can be constructed by introducing the inertial sensor. Also the inertial sensors are still not standard sensors in maritime navigation, they are drawing more and more interests due to the independency of radio signals, the shortterm high accuracy, the high output rate and the decreasing price. An Inertial Measurement Unit (IMU), which is composed of three orthogonal accelerometers and gyroscopes, can offer the navigation parameters like position, velocity, rate of turn and attitude. For this reason, introducing an IMU allows the integrity monitoring for relevant sensors. A drawback is that the IMU cannot work alone for long-term use and hence needs to be integrated with other sensors.

\subsection{RAIM}

Classic RAIM algorithms can also be applied to the maritime navigation. The only problem is the determination of the a priori measurement error for pseudoranges. This relies on standardized specification for different operation areas. If this is not available, empirical values have to be used. As an added value of the integrated system, an enhanced RAIM aided by the antenna dynamics can be implemented. The classic "snapshot" RAIM is based on the received pseudorange data of current epoch. Once the antenna dynamics can be determined, the antenna position can be predicted from the position of last epoch. This makes the pseudoranges of current epoch predictable. The predicted pseudoranges serve as additional observation to enhance the integrity monitoring. 


\subsection{Integrity monitoring for other independent navigation sensors}

Except for GNSS, the error estimation for navigation sensors is difficult as the sensor raw data is not directly processed. However, the sensor fusion algorithm makes it possible. Once the error estimation of one sensor data is available, the fusion algorithm allows the error estimation for other relevant sensors. For example, if the error of GNSS- based SOG can be properly assessed, the error analysis of speed log is also possible.

\subsection{Integrity monitoring in integration algorithm}

The plausibility tests, validity tests and compatibility tests are suitable for detecting gross sensor failure but not sensitive for slight error, time-variant errors and drifts. The Kalman filter-based algorithm could offer high sensitivity of detecting these errors. Integrity monitoring based on Kalman filter can be categorized into the following approaches [27].

\subsubsection{Kalman filter estimates (bias check)}

In a Kalman filter, the errors of navigation parameters can be estimated. If an estimated error is significantly larger than the error level specified by the manufacturer, it is likely to be a failure in the sensor.

\subsubsection{Innovation-based approaches}

The innovations indicate the consistency of the actual measurements and the measurements predicated by state estimates. Innovation filtering may be used to detect large discrepancies immediately, whereas innovation sequence monitoring enables smaller discrepancies to be detected over time.

\subsubsection{Residual-based approaches}

The above-mentioned innovation filtering and sequence monitoring can also be expanded to residuals. Residuals have a smaller covariance than innovation, making them more sensitive for error detection [27]. The only shortcoming is that the processing of residuals is not an essential part of a Kalman filter routine and needs extra computing time.

\subsubsection{Parallel solution of multiple sub-filters}

Parallel-solutions integrity monitoring maintains a number of parallel navigation solutions or subfilters, each excluding data from one sensor or radio navigation signal. Each additional navigation solution is compared with the main filter using a consistency test. A significant inconsistency indicates a fault in the sensor or signal omitted from main filter. The system output is then switched to the solution omitting the faulty sensor or signal. The main drawback lies in the increased computational burden and hence this technique is preferably used for failure identification rather than failure detection

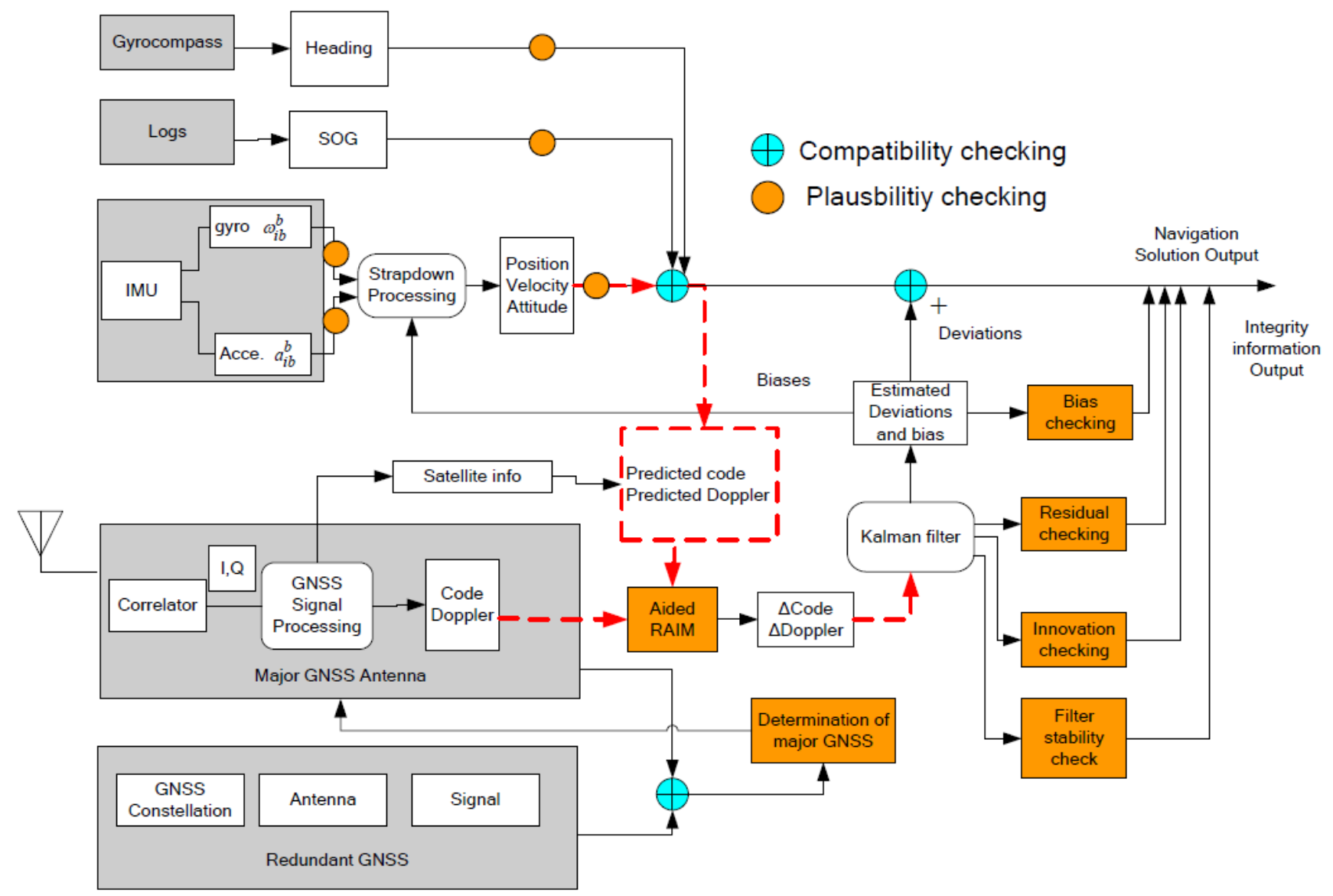

Figure 3. Integrity monitoring in the architecture of a PNT unit 


\subsection{Initial design of integrity monitoring in PNT Unit}

The major integration strategy of a PNT Unit is the integration of GNSS and IMU. An initial design of sensor integration in a PNT Unit is depicted in Figure 3. The plausibility check and compatibility check are marked at the corresponding positions in the data flow.

The plausibility check is carried out for gyrocompass, speed log and IMU output. Taking the dynamic properties of a specific ship into account, the threshold value can be derived for plausibility check.

The compatibility check is first applied to GNSS in order to determine the major GNSS antenna (also the constellation and signal, if not only one constellation or one signal is to be used). The data of major GNSS antenna will be integrated with other sensors. Both GPS and IMU offer velocity parameters and hence can be compared with speed log. IMU also outputs attitude (heading) and hence can be compared with gyrocompass.

The Kalman filter enables the bias check, innovation check and residual check. The prerequisite is a stable operation of the filter mechanism. As a stochastic system, the filter performance is based on the modeling of the observation and dynamic model, the reasonable a priori knowledge of the observation and dynamic errors, and most important, the real application scenario. It is not a trivial task to adapt the filter to all potential unexpected situations, and hence it is necessary to test the stability of the filter.

The stability test can be done either using the internal parameters of the filter, or by checking the compatibility of filter results with the results from other stand-alone sensors.

\subsection{Integrity output from a PNT Unit}

According to the previous analysis, the following integrity parameters will be supported by a PNT Unit.

TABLE 2. OUTPUT FROM A PNT UNIT

\begin{tabular}{|c|c|c|c|c|}
\hline $\begin{array}{l}\text { PNT } \\
\text { Output }\end{array}$ & $\begin{array}{l}\text { Plau- } \\
\text { sibility }\end{array}$ & $\begin{array}{l}\text { Vali- } \\
\text { dity }\end{array}$ & Compatibility & $\begin{array}{l}\text { Estimated } \\
\text { Error }\end{array}$ \\
\hline Position & $\mathrm{x}$ & $\mathrm{x}$ & $\begin{array}{l}\text { Redundant GNSS } \\
\text { Other radio-navigation } \\
\text { system }\end{array}$ & $\mathrm{x}$ \\
\hline SOG & $\mathrm{x}$ & $\mathrm{x}$ & $\begin{array}{l}\text { GNSS Doppler, Log, } \\
\text { IMU, }\end{array}$ & $\mathrm{x}$ \\
\hline $\mathrm{COG}$ & $\mathrm{x}$ & $\mathrm{x}$ & GNSS, IMU & \\
\hline Attitude & $\mathrm{X}$ & $\mathrm{X}$ & $\begin{array}{l}\text { Compass } \\
\text { multi-antenna system }\end{array}$ & $\mathrm{x}$ \\
\hline $\begin{array}{l}\text { Rate of } \\
\text { Turn }\end{array}$ & $\mathrm{x}$ & $\mathrm{X}$ & $\begin{array}{l}\text { IMU } \\
\text { heading variation } \\
\text { with time }\end{array}$ & $\mathrm{x}$ \\
\hline Time & $\mathrm{x}$ & $\mathrm{x}$ & $\begin{array}{l}\text { Other time sensors } \\
\text { internal clock of an INS }\end{array}$ & \\
\hline
\end{tabular}

This paper focuses on a maritime integrated PNT Unit as the on-board part of maritime PNT system. The aim of the PNT Unit is the robust provision of position, navigation and timing information in accordance with the performance requirements of the actual operational region. IALA has introduced the term PNT device [2] as "a device using any available IMO recognized radio navigation systems simultaneously to provide the best electronic position fix for the ship". The PNT Unit, proposed in this paper, follows a more general approach in combining available radio navigation systems and their augmentation services with other on-board PNT sensors. The core of the PNT Unit is a processing system, which combines all available PNT sensors. The PNT Unit is on the one hand part of the integrated PNT System and on the other hand part of the on-board INS.

Focusing on integrity for PNT information we have analyzed the state-of-the-art integrity monitoring approaches with respect to the identified user needs. Based on this, a demand on the development of an enhanced integrity monitoring for all relevant PNT data within an integrated PNT Unit can be deduced. Such a PNT Unit should use sensor and data fusion methods to provide PNT output data and improve integrity monitoring for these output data. For both functionalities a high degree of standardization is desired in order to achieve comparable and reliable results for their harmonized application.

Subsequently we have introduced a preliminary integrity monitoring concept for a PNT Unit which also includes additional sensors in order to deliver redundancy, backup or contingency functionality.

Finally it should be stated that this paper can only be seen as a starting point towards the realization of an integrated onboard PNT Unit for maritime applications. In a next step, after consolidation of the architecture, we plan to develop a demonstrator system of a PNT Unit.

\section{REFERENCES}

[1] IMO, NAV 54/25 Annex 12 Draft strategy for the development and implementation of e-Navigation, 2008.

[2] IALA, World Wide Radio Navigation Plan, Edition 1, 2009.

[3] IMO, Resolution MSC.112(73): Adoption of the Revised Performance Standards for Shipborne GPS Receiver Equipment, 2000.

[4] IMO, Resolution MSC.113(73): Adoption of the Revised Performance Standards for Shipborne GLONASS Receiver Equipment, 2000.

[5] IMO, Resolution MSC.114(73): Adoption of the Revised Performance Standards for Shipborne DGPS and DGLONASS Maritime Radio Beacon Receiver Equipment, 2000. 
[6] IMO, Resolution MSC.115(73): Adoption of the Revised Performance Standards for Shipborne GPS/GLONASS Combined Receiver Equipment, 2000.

[7] IMO, Resolution MSC.233(82): Adoption of the Performance Standards for Shipborne Galileo Receiver Equipment, 2006.

[8] IMO, Resolution A.915(22): Revised Maritime Policy and Requirements for A Future Global Navigation Satellite System (GNSS), 2001.

[9] IMO, NAV 56/WP.5 Development of an e-Navigation Strategy Implementation Plan, 2010.

[10] IALA, Recommendation R-129: On GNSS Vulnerability and Mitigation Measures Edition 2, 2008.

[11] IMO, SOLAS Chapter V: Safety of Navigation, 2002.

[12] R. Ziebold, Z. Dai, T. Noack, und E. Engler, "Concept for an Integrated PNT-Unit for Maritime Applications,” Proceedings of the 5th ESA Workshop on Satellite Navigation Technologies. Navitec 2010, 8.-10. Dec. 2010, Noordwijk, The Netherlands.

[13] IMO, Resolution MSC.96(72): Adoption of Amendments to Performance Standards for Devices to Measure and Indicate Speed and Distance, 2000.

[14] IMO, Resolution A.424(XI): Performance Standards for Gyro Compasses, 1979.

[15] IMO, Resolution A.382(X): Magnetic Compasses Carriage and Performance Standards, 1977.

[16] IMO, Resolution MSC.116(73): Performance Standards for Marine Transmitting Heading Devices (THDs), 2000.

[17] IMO, Resolution A.224(VII): Performance Standards for Echo- Sounding Equipment.
[18] IMO, Resolution A.526(13): Performance Standards for Rate-Of-Turn Indicators, 1983.

[19] IEC, IEC 61924 Maritime navigation and radiocommunication equipment and systems - Integrated navigation systems - Operational and performance requirements, methods of testing and required test results, 2006.

[20] B.W. Parkinson und J.J. Spiker, Global Positioning System: Theory and Applications: Volume II, American Institute of Aeronautics \& Astronautics, 1996.

[21] IEC, Maritime Navigation and Radiocommunication Equipment and Systems - Global Navigation Satellite Systems (GNSS) - Part 1: Global Positioning System (GPS) Receiver Equipment, Performance Standards, Methods of Testing and Required Test Results.

[22] IALA, Recommendation R-121: The Performance and Monitoring Of DGNSS Services in the Frequency Band $283.5-325 \mathrm{KHz}, 2004$.

[23] D. Minkwitz und S. Schlueter, "Integrity Assessment of a Maritime Carrier Phase Based GNSS Augmentation System,” in Proceedings of ION GNSS, Portland, USA: 2010.

[24] IMO, MSC.86(70): Adoption of new and amended performance standards for navigational equipment, 1998.

[25] IMO, Resolution MSC.252(83): Adoption of the Revised Performance Standards for Integrated Navigation Systems (INS), 2007.

[26] IMO, Resolution A.953(23): World-Wide Radio Navigation System.

[27] P.D. Groves, Principles of GNSS, Inertial, and MultiSensor Integrated Navigation Systems (GNSS Technology and Applications), Artech House Publishers, 2007. 